\title{
El modelo de enlace en investigación: una alternativa frente a las complejidades del saber contemporáneo
}

\section{(The connection model in research: an alternative to the complexities of contemporary knowledge)}

\author{
*Horacio Ademar Ferreyra \\ Georgia Blanas ${ }^{1}$ \\ Universidad Católica Córdoba, Córdoba y Universidad Nacional de Villa María - Argentina
}

*Email de correspondencia: hferreyra@coopmorteros.com.ar

\section{RESUMEN}

La práctica contemporánea del saber evidencia un giro en el quehacer investigativo, el cual, a lo largo de los años se ha visto condicionado por paradigmas epistemológicos y metodológicos de modelos que han separado a la ciencia de la práctica comunitaria. Bajo la idea de que el quehacer investigativo debe tener como fundamento una concepción de persona y de sociedad, a través de este trabajo se propone el modelo de enlace como un modelo alternativo en investigación. El modelo de enlace consiste en una "plataforma conceptual" que se alinea a diferentes supuestos teóricos, metodologías de trabajo, concepciones de la realidad y sentido de la intervención en el campo social y educativo, los mismos que se asientan sobre el principio de la simplificación y la complejidad como centro de construcción del conocimiento. Debido a que consideramos al modelo del enlace como una práctica, lo presentamos, siendo coherentes con su naturaleza epistémica, para ser debatido e invitar a los lectores a relacionar, disentir y converger y de esta manera, concretar cambios profundos en nuestra praxis.

Palabras clave: modelo de enlace; quehacer investigativo; revolución investigativa; saber contemporáneo.

${ }^{1}$ Se agradecen las reflexiones compartidas con los colegas e investigadores: Lic. Claudio Barbero (Subsecretaría de Estado de Promoción de Igualdad y Calidad Educativa del Ministerio de Educación de la Provincia de Córdoba), Lic. Jacinta Eberle (Universidad Católica Argentina / Delegación Paraná Argentina) y Lic. Silvia Vidales (Universidad Nacional de Córdoba) 


\begin{abstract}
The contemporary practice of knowledge reveals a shift in the research work, which, over the years has been influenced by epistemological and methodological paradigms of models that have separated the science of the community practice. Under the idea that the research work should be grounded in a conception of person and society, through this work, we propose the connection model as an alternative research model. The connection model consists of a "conceptual platform" that is aligned to different theoretical assumptions, investigation methodologies, conceptions of reality, and a sense of intervention in the social and educational field, which base on the principle of simplification and complexity as the center tituted by investigation units, categories, typologies, and integrated concepts to configure a theory of knowledge construction. Since we consider the connection model as a practice, we present it here, being consistent with its epistemic nature, for discussion and invite readers to relate, dissent and converge, and thus demand important changes in our practice.
\end{abstract}

Keywords: connection model; research work; research revolution; contemporary knowledge. 


\section{INTRODUCCIÓN}

¿Qué sería de la humanidad, si hubiese renunciado a la posibilidad de elevar su mirada más allá de la superficialidad de lo inmediato, o a la primigenia sensación que es inherente a la persona, el asombro que -en silencio- produce sorpresa y admiración, fascinación ante lo nuevo, ante la incógnita? Es ésa la fuente invisible que alimenta la búsqueda de respuestas, la insaciable necesidad de conocer. Superando lo que a sus ojos aparece como una verdad excluyente, el ser humano recupera la enseñanza que nos legara Platón en el Mito de la Caverna: osa mirar la luz, salir de la oscuridad que produce las verdades absolutas e impide que el hombre busque nuevas respuestas en la percepción de un horizonte lejano.

La conciencia despierta asombrada frente a la realidad; las preguntas esenciales, formuladas por primera vez, se erigen como punto de partida para la duda que, fundada en la contradicción, sólo parece hablar de la fragilidad de la verdad excluyente - pero a la vez inconclusa- que nos impulsa a la búsqueda de nuevos conocimientos que den otras respuestas a lo que acontece; respuestas que den sentido a nuestras dudas; dudas que no son sino expresión de nuestra necesidad de certezas... ¿̇acaso los sentidos no nos engañan o confunden con la información imprecisa que nos ofrecen? ¿no puede, acaso, nuestro dedo ser más grande que el mismo sol si sólo nos guiamos por impresiones sensibles?

Y, aun así, y frente a la lasitud de nuestras subjetividades, ¿quién nos garantiza que no nos equivocaremos ejerciendo el más escueto de los razonamientos? ¿Quién garantiza una razón infalible? Y por qué no pensar que nuestra manera de razonar podría ser, en sí misma, una nueva limitación al servicio de nuestra propia comprensión. El conocimiento construye simulacros y es diestro en operaciones complejas, en representaciones subjetivas/objetivas, en intentos de explicación, en búsqueda de respuestas, de ideales que parecieran no tener una expresión factible. Así, el pensamiento queda atrapado en imaginaciones, invenciones que aparecen como quimeras.
La búsqueda de explicación a partir del mito y la magia fue el proceso natural seguido por la humanidad, al que le sucedió la generalización que encuentra su origen en la experiencia acumulada. Las generalizaciones y su construcción a partir de la experiencia provienen de un entramado - complejo, multifacético y asociativo- de objetos, fenómenos y conceptos que tienden a hacerse estables a lo largo del tiempo y las situaciones, pasando de un proceso implícito a otro de carácter explícito. Así se hace posible dar justificación a la construcción de nuevos preceptos universales dada la apropiación que de ellos se realiza como principio o axioma, en el interjuego de la experiencia y la creencia.

Es en este proceso que se van precisando las bases del conocimiento científico y algunos conceptos, rasgos y categorías -sistemático, método científico, pensamiento crítico, universal, comprobable, razonamiento teorético, progresivo, lenguaje preciso y apropiado, explicación, posibilidad de predicción, objetividad, acumulativo, exigencia de consistencia y coherencia- demarcan el salto cualitativo que se opera desde la primigenia sensación inicial del asombro a un estadio en que la doxa es superada por el logos.

Al tomar conciencia de que la investigación se encuentra presente, de manera continua, en cada acto de la vida, y de que es fundamental observar y desplegar la curiosidad en la exploración del ámbito circundante, suele suceder que el ser humano se introduce en el camino de la búsqueda, en la construcción misma del sendero, en la estructuración de nuevos sentidos, en la visualización de diversos horizontes. Parra (2008) lo manifiesta expresamente cuando relaciona la lectura, la escritura, la investigación y precisa que un método que conduce al placer de conocer investigando supone un cúmulo de dificultades, pero también una serie de satisfacciones:

A cada instante pretendemos observar, curiosear, introducirnos en el camino o abrirnos paso si el sendero no existe. Al voltear a mirar, las cosas y los seres adquieren nuevo significado. Uno puede percibir sin mayores dificultades que existe una íntima relación entre la curiosidad infantil, el asombro, los balbuceos en la lectura de 
la realidad, el aprendizaje, la enseñanza, la escritura y la investigación. Todas ellas nos llevan a intuir la verdad, a aprehenderla, a tratar de entender, interpretar el mundo y proponer su transformación (p.8).

\subsection{La "Revolución Contemporánea del Saber"}

La "revolución contemporánea del saber ", parafraseando a Valera (2008), ha ejercido en la sociedad actual profundas transformaciones que no se reducen a la interrelación entre la producción de conocimientos científicos y las tecnologías que se generan; implican una tríada integrada que atraviesa la vida cotidiana de las personas, la ciencia y la técnica, que ya no son compartimentos estancos, sino que interactúan y se influyen mutuamente. En tal sentido, es pertinente señalar que el mayor impacto del desarrollo de la ciencia y la tecnología ha sido el cambio de sí mismas, urgente por la maduración de problemas que no encuentran solución a menos que se reconstruyan o se sustituyan dicotomías cognoscitivas y viejos ideales (Sotolongo \& Delgado, 2006, p. 28),

y más aún: "existen cambios que comienzan a retar la posición de receptor pasivo que la legitimación moderna de la ciencia confería a la vida cotidiana" (Sotolongo \& Delgado, 2006, p. 30).

\subsection{El Estado Actual de las Ciencias}

El estado actual de la producción de las Ciencias enfrenta un dilema fundado en la búsqueda de nuevas formas de producir el conocimiento, lo que Ritzer (1998) ha denominado integración y síntesis de paradigmas, y que Espina (2003) denomina "el giro epistemológico y metodológico de las ciencias". El giro epistemológico -según la autora- se caracteriza por el tránsito del paradigma de la "simplificación" al de "complejidad" en un movimiento que implica nuevos enfoques respecto del mundo y sus cualidades. El giro metodológico supone un pasaje desde las "lógicas de primer orden" -relativas a la observación de objetos- a las "lógicas de segundo orden", en las cuales se observa el objeto y los sistemas que lo observan.

Todo ello pone de manifiesto que el camino por recorrer es complejo, más aún porque sabemos que está "sembrado" de obstáculos diversos, entre los cuales merecen señalarse los siguientes: en primer término, la raigambre del positivismo que, si bien en teoría aparece como una instancia superada, está omnipresente en la práctica; a esta herencia de larga data, viene a sumarse la pugna entre los llamados teóricos sociales y los llamados prácticos sociales. Será necesario, entonces, disponer no sólo de voluntad sino fundamentalmente de una aptitud para dejar de lado los supuestos y dilemas que condicionan la producción de las Ciencias en los tiempos actuales.

\subsection{La Encrucijada de las Ciencias}

La gran encrucijada que enfrentan las Ciencias en esta revolución contemporánea del saber es poder superar los paradigmas epistemológicos y metodológicos que, si bien permitieron al conocimiento científico avances significativos, confirieron al saber un sentido atomizado, al servicio de modelos que se idearon para justificar las relaciones de control y dominación política, social y económica en desmedro del desarrollo de los pueblos y de la libertad de los seres humanos.

La revolución contemporánea del saber implica nuevas formas de producir y gestionar el conocimiento, recuperando el sentido del cambio en la formulación y el abordaje de los problemas. Para que el conocimiento científico supere las concepciones unilaterales y predominantes y los saberes descontextualizados, es necesario reconstruir el tejido entre el conocimiento científico social y el natural. Al ponerse en discusión los límites tradicionales de la ciencia, se re-plantea la cuestión de su objeto como asunto metodológico y ético y comienzan a surgir soluciones teóricas innovadoras desde nuevos enfoques: desde una nueva Epistemología.

Todo esto conlleva la necesidad de reconceptualizar las condiciones de producción y gestión de conocimiento, puesto que el ideal clásico disciplinario de la racionalidad lleva, en su propio desarrollo, a obstaculizar el conocimiento de la realidad como una totalidad (el ideal analítico presupone desmembrar el objeto como totalidad para su aprehensión de manera inmediata en sus

\section{Cumbres}


partes). Por otra parte, este saber-poder disciplinario ha tenido efectos negativos para el avance de un diálogo de saberes cada vez más transdisciplinar que viabilice un esfuerzo investigativo orientado a comprender las relaciones como totalidades complejas. El saber científico omnipotente, separado de la práctica comunitaria, ha devenido en instrumento de dominación de las personas, al situarse por encima de él. Todos estos factores han incidido en un cuestionamiento al papel y alcance de las ciencias en la actualidad.

A lo largo del tiempo, los hallazgos y contradicciones de los aportes científicos han puesto de manifiesto que el desarrollo de las ciencias está condicionado por sus propios límites. Se impone, entonces, un replanteo de las nociones de ciencia, saberes, verdad, como así también reconsiderar que el pensamiento científico se crea y construye en diálogo con la cotidianidad, concepción ausente en los modelos tradicionales. La dicotomía entre el saber teórico -aportado por la academiay el llamado saber comunitario ha vaciado a la Ciencia de sentido y, en consecuencia, la ha desnaturalizado.

Todo lo expuesto permite inferir que, en la actualidad, se está generando una "revolución contemporánea del saber" que conduce a una reflexión crítica sobre el estado actual de las ciencias y acerca del impacto que tal revolución tiene en ellas.

\section{LA INVESTIGACIÓN CIENTÍFICA}

Las diversas razones y los argumentos que se han formulado en las últimas décadas han demarcado (de manera explícita o implícita) las ideas sobre la investigación y es posible dar cuenta de procesos de alteración de estados o prácticas existentes. Dichos cambios han provocado y aún provocan encuentros y desencuentros que nos exigen el desarrollo de nuevas categorías de análisis para poder dar cuenta de las particulares características que asume hoy la realidad. Esta necesidad se manifiesta a partir de la constatación de un desfasaje acentuado entre las teorías y la realidad compleja, que se traduce en una crisis de y entre paradigmas. Así, desde distintos ámbitos y perspectivas, se expresa que tanto el paradigma de la "simplificación" como el de la "complejidad" han perdido -en sus versiones más radicalizadas- su capacidad para describir y comprender en sus múltiples dimensiones los resultados y los procesos, por lo cual estamos ante la emergencia de nuevos modelos fundados en la necesaria e insoslayable coexistencia paradigmática.

\section{HACIA LA CONSTRUCCIÓN DE NUEVOS MODELOS FUNDADOS EN LA COEXISTENCIA PARADIGMÁTICA: EL MODELO DE ENLACE COMO UNA ALTERNATIVA POSIBLE}

Entendemos que el quehacer investigativo debe tener como fundamento una concepción de persona y de sociedad y, en segundo término, debe preguntarse: ¿cómo podemos ayudar a las personas a estructurar su personalidad sin ser individualistas, egocéntricos?; ¿cómo aprender a ser y a vivir con los otros sin autoritarismo y discriminación?; ¿cómo construir y reconstruir la propia subjetividad a través del diálogo, en el diálogo y en el reconocimiento del otro como parte de la misma humanidad? Es decir, básicamente, interrogarnos acerca de qué manera podemos comunicar los saberes a la humanidad y al mundo que todos compartimos y en el que debemos reconocernos y distinguirnos.

Ahora bien, en el concierto actual del debate, creímos necesario revisar hacia dónde está dirigido, qué paradigmas lo sustentan, a fin de ofrecer un modelo alternativo, que posibilite, desde el "enlace", el desarrollo personal y social como ciudadano. Consideramos al modelo del enlace como una práctica y lo presentamos, siendo coherentes con su naturaleza epistémica, para ser debatido, para invitar a los lectores a relacionar, disentir y converger; para -a la vez- concretar cambios profundos en nuestras prácticas.

Desde principios de los años ochenta, en el campo de las Ciencias está emergiendo como alternativa una orientación paradigmática que engloba e intenta superar a la vez las formulacio- 
nes de los paradigmas antes mencionados (simplicidad y complejidad). La mayoría de las experiencias que se están desarrollando en diversos ámbitos se sustentan más bien en su coexistencia. Ante esta situación, afirmamos que están emergiendo nuevos modelos para reflexionar y accionar sobre esta realidad que nos preocupa y nos ocupa. Un modelo flexible y a la vez abierto, que pueda ser enriquecido progresivamente con los resultados de las investigaciones; que ayude a los profesionales vinculados a la investigación a valorar y comprender la multiplicidad de factores que intervienen en todo proceso, en sus diferentes contextos.

Parra (2005) en su trabajo de investigación denominado Proyecto investigativo institucional, PROIN plantea la necesidad de adentrarse, en primera instancia, en la definición íntima de modelo, no como una simple moda intelectual o como un referente que deba usarse para estar acorde con los postulados científicos, sino como una necesidad epistemológica que implica relaciones, búsqueda de la verdad, el compromiso y el empeño de un sujeto en pos de un objeto susceptible de ser investigado, conocido y transformado:

"Los agentes que investigan deben generar acciones transformativas sobre lo investigado y conceptualizar, aplicar y experimentar dentro de esa búsqueda de transformación contextual y visualización u horizonte de verdad" (p.77).

Nos interesa, en primer término, aclarar que entendemos al modelo como "visión sintética", "representación arquetípica", "plataforma conceptual", "estructura de orientación" y/o "construcción intencionada", en la que se inspiran y mantienen diferentes supuestos teóricos, metodologías de trabajo, concepciones de la realidad y sentido de la intervención en el campo social y educativo. El modelo de enlace construye su trama conceptual sobre la base del paradigma de la "simplificación" y el de la "complejidad" desde una perspectiva comprensiva y a la vez integradora, fundada en la interdependencia y la interacción y en construcción transdisciplinaria y reconciliadora. Esto supone avanzar más allá de una "única explicación", como así también dejar a un lado los intentos por encontrar justificaciones desde "un listado con los mejores aportes de los paradigmas". A nuestro juicio, estas perspectivas son parciales y dan cuenta de recortes arbitrarios en el campo epistemológico, a saber: el primero supone ver la situación con una única lente $y$, por ende, puede implicar mortalidad de certeza (Kuhn, 1971); el segundo supone una mirada global a partir de un catálogo que integra aquellos aspectos más relevantes de cada uno de los paradigmas. De lo que se trata es de dar origen a una nueva plataforma argumentativa de fundamento, que no arraiga en uno u otro paradigma, sino en el reconocimiento de las "conexiones", de la "complementariedad", de los "puntos de diálogo" e incluso de las tensiones existentes entre los aportes de ambos. De esta manera, los conocimientos se enriquecen a partir de una perspectiva trasdisciplinaria centrada en el "enlace" y que se fundamenta en la "interacción e interactividad humana"2 .

Esta nueva plataforma argumentativa de fundamento en la cual se sustenta el modelo de enlace no tiene su origen en la dimensión cuantitativa, ni en la cualitativa, sino en las prácticas investigativas situadas, a partir de las cuales se interpela tanto a la epistemología como a la metodología, y también a las categorías y prácticas lingüísticas que les son propias. La intención atiende a la comprensión de las situaciones para poder, de este modo, contribuir con su transformación.

En definitiva, y como intentamos mostrar en la Figura $1^{3}$, el modelo de enlace propuesto actúa como "puente" que conecta ambos paradigmas desde una perspectiva trasdisciplinaria, y

${ }^{2} \mathrm{El}$ concepto de "interacción" refiere a una acción que se ejerce recíprocamente entre dos o más personas en un contexto comunicativo. En cambio, la "interactividad" supone dicha interacción, pero mediada por una actividad o tarea transformadora en un contexto social (económico, político, cultural y tecnológico). Para ampliar, véase Maisonneuve, 1985.

${ }^{3}$ En la Figura 1 enuncia dimensiones generales en torno a las cuales cada paradigma señala un cierto énfasis.

\section{Cumbres}


al hacerlo, los enriquece a partir de una relación bidireccional y no jerárquica.

\section{CASOS}

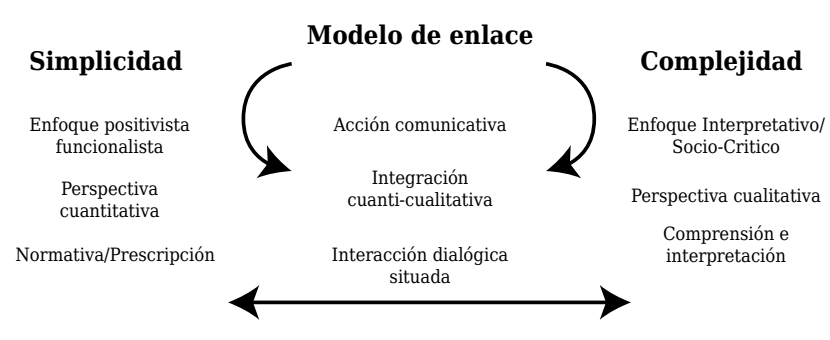

Figura 1. Modelo de enlace

A los fines de compartir con los lectores experiencias realizadas que ponen "en acto" los conceptos antes expuestos, presentamos dos casos que, según nos parece, posibilitan, enlazan y articulan, desde una perspectiva integradora, la interacción e interactividad epistemológica, metodológica y lingüística de diversas posiciones paradigmáticas con el propósito de comprender de manera inteligente y sensible la realidad en toda su complejidad. Con todo, ninguno de los casos constituye una respuesta acabada; son, por el contrario, construcciones que hacen lugar a la posibilidad de seguir pensando juntos en torno a ellas.

\section{Caso 1}

\section{Ficha Metodológica}

Estudio: Educación Secundaria en la Argentina (2000-2010). Grupo de Estudio sobre Educación Secundaria de la Facultad de Educación Universidad Católica de Córdoba (2012) ${ }^{4}$
1. AREA TEMÁTICA: Ciencias Sociales

y Humanidades

2. DISCIPLINA: Educación

3. TEMA: Educación Secundaria

4. CONTEXTO: Argentina

5. TIEMPO: 2009-2011

6. PREGUNTAS GENERADORAS

La escuela secundaria constituye, en el sistema educativo argentino, un trayecto que ha redefinido, a partir de múltiples reformas y/o transformaciones, sus objetivos y alcances, intentando responder a los requerimientos de una sociedad en permanente movimiento. Los cambios culturales, políticos, tecnológicos, económicos y sociales actuales han puesto en tensión diversos aspectos que configuran este trayecto escolar: sujetos, saberes y prácticas.

En este marco, nos hemos planteado -desde una perspectiva socio-histórica- la siguiente pregunta generadora de conocimiento: ¿Cuáles son las notas características del estado actual (2000-2010) de la Educación Secundaria argentina en el contexto latinoamericano $y$ mundial?, lo cual ha supuesto el planteo de variados interrogantes específicos:

- ¿Cuáles han sido y en qué consisten los principales cambios operados en este trayecto formativo?

- ¿Cuáles son las tensiones, problemas y temas críticos emergentes?

- ¿Qué avances se han registrado?

- ¿Qué aportes se han realizado desde la investigación educativa?

- ¿Qué experiencias institucionales y curri-

${ }^{4}$ Los integrantes del Grupo de Estudio son: Horacio Ademar Ferreyra (dirección), María Isabel Calneggia, Georgia Estela Blanas, Olga Concepción Bonetti, Laura Cecilia Bono, Sandra Liz Chiavaro, Adriana Carlota Di Francesco, María Jacinta Eberle, Gabriela Susana Haro, Marta Judith Kowadlo, Crescencia Cecilia Larrovere, Laura Delia Vargas, Blanca Laura Patricia Romero y Zulma Patricia Zárate. En el año 2011, se integraron como invitados Andrea Roxana Arnoletto, Olga Cordero, Jacqueline Mohair Evangelista, Patricia Inés Bruno, Claudia Amelia Maine, Víctor Mario Mekler, Graciela Susana Pascualetto, Marcela Alejandra Rosales, Marta Alicia Tenutto Soldevilla, Alicia Eugenia Olmos, Gabriela Cristina Peretti, Rubén Edgardo Rimondino y Silvia Noemí Vidales. También se destaca la participación de investigadores extranjeros: Ruth Catalina Perales Ponce, Lya Esther Sañudo Guerra y Martha Vergara Fregoso (México); Raquel Andrea Arias Guevara, Luz Amanda Castro Lalindes e Iván Darío Flórez Rojano (Colombia) y, recientemente, la incorporación de Gracia Navarro Saldaña (Chile). En sus inicios, el Grupo contó también con la participación de Paula Anahí Boccardi. 
culares logran dar cuenta de buenas prácticas en sus respectivos contextos?,

- ¿Cuáles son los principales retos y/o desafíos en los tiempos actuales?

\section{OBJETIVOS}

El objetivo general de la presente investigación ha sido caracterizar la Educación Secundaria Argentina en el período comprendido entre los años 2000 y 2010 para analizarla en relación con sus principales retos y posibilidades en el contexto nacional e internacional.

En cuanto a los objetivos específicos, estos son:

- Describir las transformaciones por las que ha transitado la Educación Secundaria argentina en el contexto latinoamericano y mundial.

- Relevar los aportes de investigaciones destacadas, a fin de dar cuenta de una aproximación al estado de la producción de conocimiento en torno a los problemas y propuestas vinculados con este nivel educativo.

- Analizar logros, avances, tensiones, problemas y temas críticos emergentes.

- Identificar experiencias institucionales y curriculares que logran dar cuenta de buenas prácticas en sus respectivos contextos, a los fines de delinear y sistematizar los factores que promueven la mejora.

- Enunciar propuestas de mejora para la transformación de la Educación Secundaria en la Argentina.

\section{TIPO DE ESTUDIO}

Descriptivo.

\section{METODOLOGÍA}

Mixta (cualitativa y cuantitativa).

\section{PROCEDIMIENTO}

Sobre la base de la pregunta generadora, los objetivos, el tipo de estudio y la metodología planteada, desde el punto de vista procedimental, y luego de una revisión general de la literatura sobre el tema, nos dedicamos a profundizar el estudio y delimitamos el caso a investigar.

En primer término, decidimos efectuar un estudio de alcance nacional en el contexto latinoamericano y mundial, intentando dimensionar el estado actual de la Educación Secundaria en el período 2000-2010 sin olvidar el pasado (retrospectiva) y las proyecciones futuras (prospectiva).

En segundo lugar, seleccionamos una serie de procedimientos e instrumentos considerados pertinentes para la construcción de los datos, a los fines de hacerlos consistentes desde el punto de vista metodológico.

En el caso que nos ocupa, recogimos datos relevantes de tipo cualitativo y cuantitativo a partir de la combinación y confrontación de diversas fuentes (primarias y secundarias) procedentes de distintos actores y agencias de producción del conocimiento (estatal y/o privada).

Para ello, aplicamos variadas técnicas de recolección, a saber:

a) análisis documental,

b) observaciones no participantes,

c) entrevistas semi-estructuradas y en profundidad a informantes claves,

d) entrevistas grupales,

e) seminario taller.

El trabajo documental y de campo contempló, entre otras categorías comunes de análisis, las 
siguientes : ${ }^{5}$

- currículum, saberes y prácticas en contexto,

- trayectoria escolar de los estudiantes,

- ambiente y clima institucional,

- desarrollo profesional docente,

- relaciones con la comunidad.

A los fines de la organización del trabajo y con el propósito de contribuir con la generación de conocimiento, el grupo de estudio focalizó -en una primera instancia- la indagación en cinco núcleos temáticos centrales, a saber:

a) Tendencias en las Políticas Educativas en general y de la Educación Secundaria en particular.

b) La Educación Secundaria en Latinoamérica y el mundo.

c) La Educación Secundaria en Argentina.

d) Aportes de la investigación y la producción teórica y ensayística en el campo de la Educación Secundaria.

e) Cambio, innovación y buenas prácticas en la escuela secundaria.

Por cada uno de los ejes, se designó a un integrante del grupo de estudio como responsable del proceso de sistematización de la información y producción de conocimiento. Todos, en virtud de su perfil profesional y trayectoria en la temática, aportaron su contribución a través de la participación en encuentros presenciales y virtuales que se vieron potenciados no sólo por la interacción sino por la interactividad que se generó entre los participantes.

En este marco, partimos de un análisis de tipo documental de las principales declaraciones, recomendaciones, estudios, legislación, acuerdos, investigaciones y narrativas de buenas prácticas realizadas en los últimos tiempos (2000 al 2010) ${ }^{6}$ , tanto en el ámbito internacional como el nacional y en las distintas jurisdicciones que integran la arquitectura del sistema educativo argentino. Los mismos fueron seleccionados por su actualidad, pertinencia y relevancia.

En la misma línea, se profundizó en el análisis de la producción ensayística de la última década que abordase las tensiones, problemas y temas críticos emergentes directamente vinculados con las categorías de análisis definidas. Esta tarea dio lugar a la selección de 17 producciones académicas (individuales y colectivas) de distintos especialistas de Argentina y el extranjero (México y Colombia), para ser incorporadas al Informe Final, en diálogo con los resultados del estudio.

Durante los años 2009 al 2011, asistimos como observadores no participantes a los siguientes seminarios y reuniones organizadas por el Ministerio de Educación de la Nación:

a) Ciudad Autónoma de Buenos Aires. Primer Encuentro de Trabajo Interjurisdiccional para la organización de la oferta de Educación Secundaria: 7/09/09 y 8/09/09

b) Ciudad Autónoma de Buenos Aires. Segunda Sesión de la Comisión Federal Permanente para Marcos de Referencia de la Educación Secundaria Orientada: 30/06/10 y 1/07/10.

c) Córdoba. Primer Seminario Federal para la Construcción de Marcos de Referencia para el Ciclo Orientado de la Educación Secundaria: 18/11/10 al 20/11/10.

d) La Pampa, Segundo Seminario Federal para la Construcción de Marcos de Referencia para el Ciclo Orientado de la Educación Secundaria: 14/03/11 al 16/03/11.

${ }^{5}$ Las categorías han sido construidas a partir de aportes de Kit, Alen y Terigi, 1998; Ferreyra y Peretti comps., 2006; Gobierno de Córdoba, Ministerio de Educación, 2008 y 2009.

${ }^{6}$ Se revisaron, además, materiales de la prensa gráfica y digital, así como los sitios web oficiales de los Ministerios, Consejos y/o Direcciones Generales de Nivel o de Escuelas, en el nivel nacional y jurisdiccional. Por otra parte, cabe acotar que también fueron objeto de consulta algunas publicaciones del año 2011. 
e) La Pampa. Reunión en la que se abordó el tema de Educación y Trabajo en la Educación Secundaria: 15/03/11.

f) Ciudad Autónoma de Buenos Aires. Reunión de la Comisión Federal Permanente para Marcos de Referencia: 27/04/11 al 28/04/11.

g) Ciudad Autónoma de Buenos Aires. Reunión Nacional Construcciones curriculares para Educación Secundaria. Estado de avance y proyecciones: 30/11/11.

Además, participamos del Seminario Internacional "La escolarización de los adolescentes: desafíos culturales, pedagógicos y de política educativa", organizado por el IIPE-UNESCO Sede Regional Buenos Aires en la Ciudad Autónoma de Buenos Aires desde el 14/9/11 al 15/9/11.

En dichos seminarios y reuniones participaron Ministros, Secretarios y Subsecretarios de Estado, Directores de Educación Secundaria, técnicos, supervisores, directivos, docentes y estudiantes, entre otros. En estas instancias, se desarrollaron actividades plenarias y en comisiones por temas, en las que se concretaron exposiciones de especialistas, rondas de discusión y trabajos colectivos de los participantes.

La observación no participante referida se concretó con la finalidad de recuperar -desde la perspectiva de los diversos actores- los avances, dificultades y cuestiones pendientes sobre la implementación de los distintos Ciclos que conforman la Educación Secundaria Orientada. Además, durante el transcurso de estos eventos, realizamos entrevistas no estructuradas a actores claves de las distintas jurisdicciones. Ambas intervenciones estuvieron orientadas a enriquecer el análisis documental.

Por otra parte, y siempre con la intención de enriquecer el proceso de construcción de conocimiento productivo, y de ratificar, rectificar, complementar y/o ampliar la información sistematizada, concretamos:

a) 6 consultas a especialistas nacionales e internacionales. b) 20 entrevistas abiertas no estructuradas, 4 entrevistas en profundidad a informantes claves funcionarios, investigadores, técnicos, supervisores, directivos y docentes.

c) Selección de 10 buenas prácticas tomadas de fuentes secundarias y actualizadas a partir de un trabajo cooperativo con los responsables (directivos y/o docentes).

d) 1 Seminario/taller "La Educación Secundaria en la Argentina (2000 - 2010). Una aproximación a su estudio desde distintas dimensiones (Primeros avances)" 18/11/201, con la participación de los integrantes del grupo de estudio, responsables de las buenas prácticas seleccionadas, funcionarios del Ministerio de Educación de la República Argentina y provinciales (La Pampa, Córdoba, Tucumán, Entre Ríos, provincia de Buenos Aires y Ciudad Autónoma de Buenos Aires) y expertos invitados de la Organización de Estados Iberoamericanos Sede Buenos Aires, la Universidad Católica de Santa Fe, la Universidad de Concepción de Chile y la Universidad Santo Tomás de Aquino de Colombia (un total de 38 participantes).

e) Consultas específicas a funcionarios y técnicos del Ministerio de Educación de la República Argentina (DINIECE), Ministerio de Educación de Córdoba -Subsecretaría de Promoción de Igualdad y Calidad Educativa-, Ministerio de Educación de Chubut, Dirección General de Cultura y Educación de la Provincia de Buenos Aires, Consejo General de Educación de Entre Ríos, Ministerio de Educación de la Rioja, Ministerio de Educación de Salta, Ministerio de Cultura y Educación de Misiones, Consejo Provincial de Educación de Santa Cruz, Ministerio de Educación de Tucumán, Ministerio de Educación de Río Negro y Ministerio de Educación de Santa Fe.

f) Consultas a especialistas de UNESCO-Oficina Internacional de Educación, Ginebra, Suiza (Programa para la Construcción de Capacidades); Instituto Internacional de Planeamiento Educativo IIPE-UNESCO Sede Regional Buenos Aires, Universidad Nacional de Córdoba, Universidad Autónoma de Entre Ríos, Universidad Católica de Santa Fe, Universidad Santo Tomás de Aquino de Colombia (Unidad de Investigación), Instituto

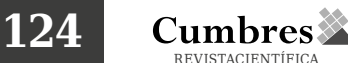


Superior de Investigación y Docencia para el Magisterio (ISIDM) y del Consejo Interinstitucional de Investigación Educativa de la Secretaría de Educación de Jalisco (México).

Los actores nacionales (de diversas jurisdicciones) y los especialistas internacionales e informantes claves han sido escogidos por su conocimiento sobre el tema, participación en los procesos de transformación, estatus en el sistema educativo y disposición para cooperar con este proyecto.

A medida que fuimos concretando avances en el trabajo documental y de campo, la sistematización de cada núcleo se fue consolidando. En esta etapa, se efectuaron múltiples intercambios entre los integrantes del grupo e investigadores externos, a los fines de avanzar en cuestiones conceptuales y metodológicas. Dicha acción se vio enriquecida con su participación -así como la de los informantes claves, técnicos, funcionarios, directivos y/o docentes responsables de las buenas prácticas seleccionadas e integrantes del grupo de estudio- en el seminario taller en el que se compartieron los avances por núcleo y se debatieron diversos aspectos a los fines de enriquecer el trabajo ${ }^{7}$.

En el transcurso de las discusiones que tuvieron lugar en el marco de las tareas propias de la sistematización de la información, surgieron algunas limitaciones de carácter teórico, producto de las diferentes formas de entender la Educación Secundaria en el contexto actual y de la disparidad de perfil y trayectoria en la investigación de cada uno de los integrantes del grupo de estudio, que provienen de diferentes campos del conocimiento (Educación, Ciencias de la Co- municación, Ciencias Políticas, etc.). También constituyeron dificultades significativas el escaso tiempo disponible de algunos de los miembros del grupo que, en su mayoría, ha podido destinar entre 6 y 24 hs mensuales al proyecto, así como el reducido apoyo financiero para el desarrollo de las actividades. En el primer caso, se desplegaron estrategias de trabajo y encuentro (presencial y virtual) que permitieran una optimización del aprovechamiento del tiempo disponible. En cuanto a las limitantes económicas, se fueron resolviendo a partir de las gestiones realizadas por los integrantes del grupo y con el apoyo de la Facultad de Educación de la UCC, del Ministerio de Educación de la Nación y de las distintas jurisdicciones que participaron de las diversas instancias.

Finalmente, se conformó, en el seno del grupo de estudio, un equipo que ha tenido a su cargo la elaboración del informe final de investigación a partir de lo sistematizado en relación con cada núcleo y en consulta con los demás integrantes, a los fines de validar y consolidar la producción.

Fuente: Ferreyra, H. (2012a). Entramados, análisis y propuestas para el debate. Aproximaciones a la Educación Secundaria en la Argentina (2000-2010). Córdoba, Argentina: Editorial Comunicarte.

\section{Caso 2}

\section{Ficha Metodológica}

Estudio: Educación Permanente de Personas Jóvenes y Adultas en la República Argentina. El caso de las provincias de Buenos Aires, Córdoba, Mendoza, Santa Fe y Tucumán (2006-2008). Posdoctoral en Ciencias Sociales (Sociedad y Educación) Universidad Autónoma Metropolitana

${ }^{7}$ De este modo, se inició, en el ámbito del grupo de estudio, tal como sostiene Gibbons, Lomoges, Nowotny, Schwartzman, Scott, \& Trow (1997), una forma de construir conocimiento diferente de la tradicional, que "supone la existencia de diferentes mecanismos para generar conocimiento y para comunicarlo, más actores procedentes de disciplinas diferentes y con historiales distintos, pero, por encima de todo, lugares diferentes donde se produce el conocimiento" (p. 31). En este caso, se pusieron en contacto diversos actores con diferentes trayectorias y procedencias institucionales, y se integraron también al proceso algunos informantes claves. 
de México, Unidad Xochimilco, División Ciencias Sociales y Humanidades (2009).

\section{A. AREA TEMÁtICA: Ciencias Sociales y Humani- dades \\ B. DISCIPLINA: Educación \\ C. TEMA: Educación de Jóvenes y Adultos. \\ D. CONTEXTO: Argentina (Provincias de Buenos Aires, Córdoba, Mendoza, Santa Fe y Tucumán). E. TIEMPO: 2007-2009}

Realizar hoy una mirada a la EPJA a partir del análisis de las políticas, nos posibilita aprovechar una oportunidad trascendental, única, necesaria y ajustada a lo que demanda la realidad socio histórica presente de la educación argentina, en el contexto internacional.

Reflexionar sobre esta trayectoria educativa nos ha implicado, en primer término, detectar una situación crítica, problemática, como es -en este caso- el déficit en la sistematización de la información y producción del conocimiento en lo que respecta a la EPJA, tanto en el ámbito nacional como provincial, lo cual dificulta los procesos de toma de decisiones de política educativa para el sector. Asumimos que este problema constituye -según nuestro criterio- la oportunidad de que se abran nuevas perspectivas en el campo de lo político. Al decir de Arendt (1996):

Una crisis nos obliga a volver a plantearnos preguntas y nos exige nuevas o viejas respuestas, pero, en cualquier caso, juicios directos. Una crisis se convierte en un desastre sólo cuando respondemos a ella con juicios preestablecidos, es decir con prejuicios. Tal actitud agudiza la crisis y, además nos impide experimentar la realidad y nos quita la ocasión de reflexionar sobre lo que esa realidad brinda (p.186).

En definitiva, lo que se encuentra en tensión es la capacidad, o no, de plantearse un problema, el aplicar o no un razonamiento que no quede atrapado en los conocimientos ya existentes; significa aquí ser crítico de aquello que nos sostiene teóricamente, o sea, ser capaces de distanciarnos de los conceptos que manejamos, así como también cuestionar lo empírico, la realidad observa- da, porque esto puede no ser lo relevante, puede ser solo el tema... En el mejor de los casos, puede ser un tema sobre el cual problematizar, para lo cual hay que estar dispuesto desde la investigación a ver qué hay más allá de la región visible de los fenómenos (De Souza, 2004, p.18), qué hay detrás de ese tema, apelando al pensamiento crítico y creativo (De Souza, 2004 y Zemelman, 2002).

Es así que observando con mayor atención la cuestión que nos ocupa, en la actual situación crítica de la EPJA lo que aparece como un cuestionamiento radical es la pérdida del hilo conductor, la ausencia de fines, del sentido hacia el cual orientarla como hecho social. Al decir de Borges (1993), "el hilo se ha perdido; el laberinto se ha perdido también. Ahora ni siquiera sabemos si nos rodea un laberinto, un secreto cosmos, o un caos azaroso. Nuestro hermoso deber es imaginar que hay un laberinto y un hilo" (p. 391), no solo para transitar estos tiempos y espacios, sino para ampliar la mirada y provocar una ruptura epistémico-metodológica en las formas teórico-prácticas, generalmente aceptadas, que actúan como guías de acción y reflexión en la comprensión de los fenómenos socioeducativos (Bourdieu, Chamboredon, \& Passeron, 1975).

En la actualidad, nos encontramos ante una crisis de los principales paradigmas (Kuhn, 1995) que han sido y son comúnmente aceptados (empírico analítico, interpretativo y crítico) en el campo de las ciencias humanas. Por lo tanto, un paradigma, como representación de una determinada perspectiva, no se convierte en "el paradigma", la única manera de ver y comprender la realidad, sino que se parte de la necesaria e inevitable coexistencia de ellos en el proceso de investigación educativa.

La aceptación o adhesión a un paradigma no lleva necesariamente al reemplazo de uno por otro, porque si esto ocurriera estaríamos reduciendo su complejidad a una sola mirada, lo que nos traería mortalidad de certezas y en su defecto, cierta parálisis paradigmática.

En los complejos procesos actuales de transformación de la EPJA, estamos convencidos de la

\section{Cumbres}


necesidad de abordar la investigación a partir de una coexistencia paradigmática dialógica, que posibilite la aproximación a la realidad objeto de estudio a través de un arsenal de métodos diversificados (cuantitativos y cualitativos), que nos permita la obtención de datos mutuamente complementarios (Vasilachis, 1993).

\section{F. OBJETIVOS}

El objetivo general de la presente investigación ha sido construir una línea de base con información confiable del Subsistema de EPJA de la República Argentina en general y de las provincias seleccionadas en particular, con el propósito de identificar las prioridades que se deberían considerar en el diseño y gestión de un plan de desarrollo socio-educativo para la Modalidad, cuya finalidad es la de incluir y mejorar los procesos y resultados en el marco de la educación permanente.

En cuanto a los objetivos específicos, estos son:

- Describir el marco socio-histórico normativo de la EPJA.

- Caracterizar la situación de la EPJA en los aspectos vinculados a las Instituciones, estudiantes, docentes y currículum.

- Identificar las iniciativas y experiencias innovadoras que se están llevando a cabo en las provincias seleccionadas, en el marco de las políticas nacionales.

\section{G. TIPO DE ESTUDIO}

Con respecto al tipo de estudio, hay dos factores que influyen a la hora de definir el tipo de investigación: el conocimiento actual del tema a investigar y el enfoque que pretenden darle al estudio los investigadores (Hernández, Fernández, \& Baptista, 2003). En cuanto al conocimiento actual sobre el tema que nos ocupa, como ya se expresó, no hay mayores abordajes teóricos de este problema. La mayoría de los trabajos se ha realizado en el ámbito de la educación general básica y en el campo social y de la salud desde perspectivas más bien cuantitativas. Es por ello que, sobre la base de lo anterior, optamos por una investigación de tipo descriptiva con un enfoque mixto (cuantitativo y cualitativo), convencidos de la necesidad de explicitar las múltiples interacciones dinámicas que se dan en la realidad, asistidos en cada caso por dispositivos metodológicos adecuados que triangulen datos cuantitativos y cualitativos.

\section{H. POBLACIÓN Y MUESTRA}

La población objeto de este estudio es el Subsistema de EPJA (Nivel Primario y Secundaria), del cual hemos seleccionado como muestra a las provincias de Buenos Aires, Córdoba, Santa Fe, Mendoza y Tucumán por ser las que concentran el mayor número de Unidades Educativas y Población Estudiantil (más del 50\% del país) (Red Federal de Información Educativa, DINIECE, Ministerio de Educación, Ciencia y Tecnología de la Nación, 2006) y las que han realizado innovaciones en los últimos años 2006-2008. Por otra parte, por tener posibilidad de acceso a fuentes de información y estadísticas.

\section{PROCEDIMIENTO}

Sobre la base del problema de investigación y los objetivos planteados, desde el punto de vista procedimental, y luego de una revisión general de la literatura sobre el tema, nos dedicamos a profundizar el estudio y delimitamos el caso a investigar.

En primer término, decidimos efectuar un estudio en dos planos: uno federal y otro focalizado en las provincias seleccionadas, intentando dimensionar, de ese modo, el problema de la producción y uso de la información en el campo de la EPJA.

En segundo lugar, seleccionamos una serie de procedimientos e instrumentos considerados válidos para la obtención de los datos, a los fines de hacerlos consistentes desde el punto de vista metodológico.

En el caso que nos ocupa, recogimos datos re- 
levantes de tipo cuantitativo y cualitativo a partir de la combinación y confrontación de diversas fuentes (primarias y secundarias) procedentes de distintos actores y escalas del sistema educativo (internacional, nacional y provincial).

Para ello, aplicamos variadas técnicas de recolección (observaciones no participantes, entrevistas semi-estructuradas y en profundidad) y un amplio procedimiento en la consulta de información producida en otros ámbitos oficiales y académicos, tanto en el nivel nacional como provincial.

Partimos de un análisis de tipo documental de las principales declaraciones, recomendaciones, estudios, legislación, acuerdos e investigaciones realizadas en los últimos tiempos, tanto en el ámbito nacional, como en el de las distintas jurisdicciones que integran la arquitectura del sistema educativo argentino. De igual modo también fueron analizados los datos estadísticos suministrados por el Ministerio de Educación de la Nación y las áreas estadísticas de las provincias involucradas. Los mismos fueron seleccionados por su actualidad, pertinencia y relevancia. Los dispositivos que fueron considerados fuentes primarias y sobre los cuales efectuamos el análisis, son:

\section{Cuadro 11. Documentos primarios ${ }^{8}$}

- Consejo General de Educación (CGE) (2007). I Encuentro Nacional y II Provincial de Experiencias Educativas Innovadoras, organizado por el CGE. Dirección de Educación de Jóvenes y Adultos de la Provincia de Entre Ríos, Argentina.

- DINIECE. Anuario Estadístico 2005, 2006 Y 2007.

- Ferreyra, H. y Blanas, G. (2009). La construcción de una línea de base en el campo de la educación de personas jóvenes y adultas en la República Argentina 2006. Córdoba, Argentina. Mímeo
- Kit, I. (2007). Evolución reciente y situación actual de la Educación de Adultos en Argentina 1997-2005. Educación para todos Asociación civil. Argentina. Recuperado de http://www.educacionparatodos. org.ar/conocer/Tendencias_actual_de educacion_de_adultos.pdf

- Ministerio de Trabajo, Empleo y Seguridad Social (MTEySS) (2007). Informes de Organismos Provinciales y de Gerencias de Empleo y Capacitación Laboral. Documento de trabajo. Argentina: SE. DNOyFP.

- Ministerio de Trabajo, Empleo y Seguridad Social (MTEySS). (2008). La educación como política de empleo en la argentina. El caso de la educación de jóvenes y adultos en las Provincias de Buenos Aires, Córdoba, Mendoza, Santa Fe y Tucumán. (Consultor Dr. Horacio Ferreyra). Argentina: Dirección Nacional de Orientación y Formación profesional. Subsecretaría de Políticas de Formación Profesional y Empleo MTEySS.

- Rodríguez, L. (2008). Situación presente de la Educación de Jóvenes y Adultos en Argentina. México: CREFAL. Recuperado de http://www.crefal.edu.mx/descargas/ informes_nacionales/argentina.pdf

Durante los años 2006 y 2007, asistimos como observadores no participantes a un taller y a tres encuentros tecnopolíticos con directivos provinciales y responsables de la EPJA de las distintas jurisdicciones del país (RT), a saber:

\section{Cuadro 12. Reuniones Técnico-Políticas (RT)}

- Taller "Bases para un Proyecto de Formación a lo largo de la vida". Organizó: MTEySS. Ciudad de Buenos Aires. 14 y 15 de Agosto de 2006. Participación: 39 personas.

- II Encuentro Nacional “La formación como

\footnotetext{
${ }^{8}$ Con posterioridad a la finalización del estudio y a los fines de enriquecer y actualizar la presente publicación, se consultaron las siguientes fuentes primarias: Schoo, Sinisi y Montesinos: 2010; De la Fare, 2010 y 2011; Relatorías del MTEYSS, 2010 y 2011.
}

\section{Cumbres}


política de Empleo. Ejes para un aprendizaje a lo largo de toda la vida". Córdoba, Argentina, del 20 al 22 de noviembre de 2006. Organizó: MTEYSS y MECYT de la Nación. Participación: 150 actores de 21 jurisdicciones.

- Encuentro de experiencias exitosas: "Articulación Cruzada". MTEySS. Mendoza, 28 de junio de 2007. Participación: 35 actores de las provincias de Mendoza, La Pampa, Entre Ríos, Río Negro, Jujuy, Buenos Aires.

- III Encuentro Nacional "La formación como política de Empleo. Consolidar la articulación intersectorial". Mar del Plata, Argentina, del 5 al 7 de noviembre de 2007. Organizó: MTEYSS y MECYT de la Nación. Participación: 250 actores del campo de la educación, trabajo y producción de 23 jurisdicciones.

En los mencionados eventos se desarrollaron actividades plenarias y en comisiones por temas, en las que se concretaron exposiciones de especialistas, talleres y socialización de experiencias jurisdiccionales. Dicha observación no participante se concretó con la finalidad de recuperar, desde la perspectiva de los actores, los avances, dificultades y cuestiones pendientes sobre la implementación de los distintos ciclos y trayectos que conforman la EPJA en las distintas jurisdicciones, a fin de enriquecer el análisis documental.

Por otra parte, efectuamos 24 entrevistas abiertas no estructuradas a funcionarios, técnicos, supervisores, directivos y docentes y 6 entrevistas en profundidad a investigadores (IC) del Ministerio de Educación Nacional y de las distintas provincias, con el propósito de completar y/o corroborar la información sistematizada sobre el estado de situación de la EPJA. Los informantes claves han sido escogidos por su conocimiento sobre el tema, participación en los procesos de transformación, status en el sistema educativo y disposición para cooperar con este proyecto.

Ambas miradas, la documental y la de campo (incluido el diseño de los protocolos de observación y encuestas), las efectuamos teniendo en cuenta las siguientes categorías de análisis:

- instituciones educativas (cantidad, infraestructura, equipamiento, normativa),

- estudiantes (edad, sexo, características socio-culturales, trabajo, eficiencia interna, egreso, certificaciones),

- docentes (directivos, profesores, nivel de formación inicial y continua),

- currículum (contenidos, modalidades de enseñanza, prácticas educativas, vinculación de la educación con el trabajo y la vida),

- iniciativas y experiencias significativas.

En el proceso de triangulación, el registro se efectuó en la siguiente matriz de sistematización (ver Tabla 1):

En todo este procedimiento, nos hemos aproximado, simultáneamente, a las manifestaciones más visibles y concretas de la EPJA y al mundo de los significados de las acciones y relaciones que allí se construyen y reconstruyen en interacción con el contexto. Esto nos permite afirmar desde lo metodológico que el conjunto de datos cuantitativos y cualitativos no se oponen, sino que se complementan en la interpretación de la realidad alcanzada por ellos.

Tabla 1. Matriz de sistematización

\begin{tabular}{|c|c|c|c|c|c|}
\hline \multirow[b]{2}{*}{$\begin{array}{l}\text { Categorias } \\
\text { Varisble }\end{array}$} & \multicolumn{2}{|l|}{ Entrevistas } & \multirow[b]{2}{*}{ Observación } & \multirow[b]{2}{*}{$\begin{array}{l}\text { Información } \\
\text { Estadistica }\end{array}$} & \multirow[b]{2}{*}{$\begin{array}{l}\text { Informes de } \\
\text { Investigneión }\end{array}$} \\
\hline & $\begin{array}{l}\text { Semi- } \\
\text { estructurada }\end{array}$ & Profundidad & & & \\
\hline \multicolumn{6}{|l|}{$\begin{array}{l}\text { Iestifución } \\
\text { Educativa }\end{array}$} \\
\hline \multicolumn{6}{|l|}{ Estudiagtes } \\
\hline \multicolumn{6}{|l|}{ Docente } \\
\hline \multicolumn{6}{|l|}{ Currolculum } \\
\hline \multicolumn{6}{|l|}{$\begin{array}{l}\text { Iniciativas y } \\
\text { experiencias }\end{array}$} \\
\hline \multicolumn{6}{|l|}{ Emergentes } \\
\hline$\ldots . .$. & & & & & \\
\hline
\end{tabular}

Fuente: Ferreira y Blanas (2016) 


\section{REFLEXIONES FINALES}

El proceso investigativo como interacción social enmarcada en una cultura, situada en un contexto, con normas, con prescripciones que responden a una visión científica, a paradigmas -que, en muchos casos, resultan poco claros y a veces hasta se contraponen- tiene como meta promover la construcción de subjetividades únicas, personas socialmente integradas, ciudadanos (no sólo habitantes) que puedan interactuar con su mundo y posicionarse desde un modelo elegido. Tales metas requieren de procesos de construcción, producción, comunicación y circulación del conocimiento que pongan en diálogo lo simple y lo complejo, las dimensiones cuantitativas con las cualitativas, la prescripción y la norma, los aportes tradicionales y las perspectivas críticas, al servicio del desarrollo humano. En este sentido, el modelo de enlace se constituye en una alternativa que favorece el paso de la interdisciplinariedad y la simplicidad a la transdiciplinariedad y la complejidad, enfatiza el carácter multirreferencial y multideterminado de los fenómenos y supone un sujeto reflexivo, implicado en la relación con lo que conoce, transformado y transformador en la producción de conocimiento.

Para construir, comunicar y compartir conocimiento de esta manera, se requiere de investigadores y de docentes reflexivos, que interpreten y comprendan no sólo la ciencia que enseñan sino y simultáneamente, la realidad de cada estudiante, del contexto, de la historia y de la ética que promueve el bien común. Investigadores y docentes con espíritu de indagación y búsqueda, ya que -como afirma Parra (2008)

... tanto el docente como el investigador se detienen a observar el mundo circundante y hacen conjeturas, ¿quién no las hace?, ¿por qué se hacen? Sencillamente porque el hombre, debido a su naturaleza cuestionante, a su querer explicar el mundo, a su capacidad de asombro, a sus posibilidades de discernimiento y desacuerdo, observa la realidad, contrasta las variables, revisa los estudios y los puntos de vista que otros tienen y se aventura a plantear posibilidades, a trazar horizontes, a proponer, a establecer posibles re- laciones, aún en hechos aislados, a tratar de explicar fenómenos (p.250).

Esta propuesta justifica, esencialmente, una actitud de provocación para generar cambios con ciencia y conciencia, como disparador para que como profesores e investigadores no nos movamos sólo por la intuición, por ideales u objetivos enunciados por otros (sólo como obedientes sumisos de normas pre-establecidas), sino que seamos capaces de interrogarnos: ¿qué propósitos efectivamente hemos concretado?; ¿qué procesos y resultados integrales hemos conseguido?; ¿en cuánto hemos promovido la construcción de la subjetividad?

Sólo de esta manera se abre ante nosotros un camino, lleno de esperanza y de utopías colectivas como lo es este de jugar investigando, recuperando la importancia que ejercen las sensaciones en la persona; recuperando el sentido primigenio del quehacer investigativo. En la actualidad, las estrechas interrelaciones de los elementos naturales y las que ellos establecen con el mundo social han adquirido un grado de complejidad tal que dificulta la comprensión de los problemas derivados de ellas. Por eso, hay que atreverse -del mismo modo que propone Perkins (2010) para el aprendizaje- a "jugar el juego completo" en la construcción del conocimiento, ese caudal inagotable de información, experiencias e ideas capaces de hacernos comprender la compleja, dinámica y cambiante trama de la realidad.

En la actualidad, la industria del conocimiento pone de manifiesto la necesidad de recuperar el sentido de la humanización, la personalización y la socialización entre personas, pueblos y naciones. Sin duda, este constituye un proceso que implica interrelación como un acto de revelación en el que un ser humano se manifiesta, se hace presente ante otro para enseñarle, para mostrarle algo de su propio ser, para ayudarlo a descubrirse a sí mismo. Este modo de entender el quehacer investigativo es un reto en la sociedad actual en la que, en muchas ocasiones - y a pesar de los progresos científicos, tecnológicos, comunicacionales-, las relaciones interpersonales se encuentran resquebrajadas (Ferreyra \& Caelles, 2010).

En síntesis, lo que postulamos es un conoci-

\section{Cumbres}


miento que permita ser compartido por todos los pueblos si se quiere hablar de equidad. Entendemos que la calidad de vida de las personas depende cada vez más del conocimiento científico y tecnológico disponible y, por ello, el acceso al mismo se ha transformado en uno de los pilares de esa sociedad que queremos. En tiempos en los que el desarrollo sostenible se presenta como la única opción lógica y coherente, la difusión y divulgación científica debe realizar una importante contribución a la transformación de la sociedad. El éxito se alcanzará cuando se logre construir el puente entre la investigación y la comunidad, paso esencial para que la ciencia se vuelva parte de la cultura.

La democratización del conocimiento y de la ciencia, cuya tarea ha sido encomendada prioritariamente a las Universidades, y a la cual es primordial que se integren otras instituciones y organizaciones responsables del desarrollo humano y social, es una empresa que no ha sido aún cumplida...Nos corresponde, sigamos en camino.

\section{REFERENCIAS BIBLIOGRÁFICAS}

Arendt, H. (1996). Entre el pasado y el futuro: Ocho ejercicios sobre la reflexión política. Barcelona, España: Península.

Argentina, Ministerio de Trabajo, Empleo y Seguridad Social (MTEySS). (2010). Relatoría. Encuentro Nacional de Educación y Trabajo: "La formación continua como herramienta estratégica para la inserción en el trabajo". Buenos Aires: Dirección Nacional de Orientación y Formación profesional. Subsecretaría de Políticas de Formación Profesional y Empleo MTEySS.

Argentina, Ministerio de Trabajo, Empleo y Seguridad Social (MTEySS). (2011). Relatoría. Seminario Taller "Propuestas innovadoras en la Educación de Jóvenes y Adultos. Facilidades para el acceso, la permanencia y la certificación de estudios formales". Buenos Aires: Dirección Nacional de Orientación y Forma- ción profesional. Subsecretaría de Políticas de Formación Profesional y Empleo MTEySS.

Böhm, W. (1991). Teoría y praxis. México DF: OEA. UCC.

Borges, J. (1993). Obras completas. Barcelona, Espana: Círculo de Lectores.

Borón A. (2006). La revolución contemporánea del saber y la complejidad social. Buenos Aires: CLACSO.

Bourdieu, P., Chamboredon, J., \& Passeron, J. (1975). El oficio del sociólogo. México DF: Siglo XXI.

De la Fare, M. (2010). Principales ideas, discusiones y producciones en Educación de Jóvenes y Adultos en Argentina: aportes para una reconstrucción histórica: Aportes para una reconstrucción histórica. Buenos Aires: Ministerio de Educación de la Nación. DINIECE.

De la Fare, M. (2011). Investigaciones y estudios en torno a la Educación de Jóvenes y Adultos en Argentina: Estado del conocimiento. Buenos Aires: Ministerio de Educación de la Nación. DINIECE.

De la Herrán, A. (2003). El nuevo paradigma Complejo-Evolucionista en Educación. Revista Complutense de Educación, 12 (2), 499-592.

De Souza, M. (2004). Investigación social: teoría, método y creatividad. Buenos Aires: Lugar Editorial.

Espina, M. (2003). Complejidad y pensamiento social. En L. Carrizo, M. Espina, \& J. Klein, Transdisciplinariedad y Complejidad en el Análisis Social (pp.13-36). Montevideo: UNESCO. Recuperado de http://en.scientificcommons. org/8830067

Ferreyra, H. (2012a). Apéndice Metodológico. En Entramados, análisis y propuestas para el de- 
bate. Aproximaciones a la Educación Secundaria en la Argentina (2000-2010). Córdoba, Argentina: Facultad de Educación Universidad Católica de Córdoba.

Ferreyra, H. (2012b). Apéndice. En Educación de Jóvenes y Adultos: políticas, sujetos y contextos (pp.107-114). Buenos Aires: Novedades Educativas.

Ferreyra, H., \& Caelles, A. (2010). Más allá del paradigma del consenso y del conflicto: el modelo del encuentro educativo. Revista Magistro, 3(5), 49-57.

Ferreyra, H., \& Pedrazzi, G. (2006). Teorías y enfoques psicoeducativos del aprendizaje. Aportes conceptuales básicos: El modelo de enlace para la interpretación de las prácticas escolares en contexto. Buenos Aires: Novedades Educativas.

Ferreyra, H., \& Peretti, G. (2006). Diseño y gestión de una Educación Auténtica. Buenos Aires: Novedades Educativas.

Gibbons, M., Lomoges, C., Nowotny, H., Schwartzman, S., Scott, P., \& Trow, M. (1997). La nueva producción del conocimiento. La dinámica de la ciencia y la investigación en las sociedades contemporáneas. Barcelona, España: Pomares - Corredor.

Gobierno de Córdoba. Ministerio De Educación. Secretaría De Educación. Subsecretaría de Promoción de Igualdad y Calidad Educativa (2008). Orientaciones sociopedagógicas para la construcción de una propuesta institucional de retención e inclusión con calidad. Nivel Medio. Córdoba, Argentina: Autor.

Gobierno de Córdoba, Ministerio de Educación. Secretaría de Educación. Subsecretaría de Promoción de Igualdad y Calidad Educativa (2009). Discusión sobre la Educación Secundaria Provincia de Córdoba. Informe 2. Problemas y propuestas desde las investigaciones.
Córdoba, Argentina: Autor.

Habermas, J. (1998). Teoría y Praxis. Madrid: Atalaya.

Hernández, R., Fernández, C., \& Baptista, P. (2003). Metodología de la Investigación. México DF: McGraw-Hill.

Kit, I., Alen, B., \& Terigi, F. (1998). Propuesta pedagógica institucional: Plan Social Educativo. Buenos Aires: Ministerio de Educación de la Nación.

Kuhn, T. (1995). La estructura de las revoluciones científicas. México DF: FCE.

Maisonneuve, J. (1985). La dinámica de los grupos. Buenos Aires: Nueva Visión.

Morin, E. (1999). Los siete saberes necesarios para la educación del futuro. París: UNESCO.

Morin, E. (2003, Enero 15). Globalización, civilización y barbarie. Diario Clarín, pp. 16, 17.

Parra, O. (2005). Proyecto investigativo institucional, PROIN. Un enfoque para la concepción y el desarrollo de la investigación. Bogotá: Universidad Santo Tomás.

Parra, O. (2008). El placer de investigar. Bogotá: Universidad Santo Tomás.

Parra, O. (2009). Las líneas: elementos determinantes de la investigación. Hallazgos, (11), 15 -32 .

Parra, O. (2011). El placer de conocer investigando: Gestión del conocimiento. Bogotá, Colombia: Universidad Santo Tomas.

Perkins, D. (2010). El aprendizaje pleno: Principios de la enseñanza para transformar la educación. Buenos Aires: Paidós.

Red Federal de Información Educativa, DINIECE,

\section{Cumbres}


Ministerio de Educación, Ciencia y Tecnología de la Nación (2006). Anuario Estadístico Educativo 2006. Buenos Aires: Autor. Recuperado de http://goo.gl/GXHeBg

Ritzer, G. (1998). Teoría sociológica contemporánea. Madrid: Mc. Graw-Hill.

Sander. B. (1990). Educación, administración y calidad de vida. Buenos Aires: Santillana.

Schoo, S., Sinisi, L., \& Montesinos, M. (2010). Trayectorias socio-educativas de jóvenes y adultos y sus experiencias con la escuela media. Buenos Aires: Ministerio de Educación de la Nación. DINIECE.

Sotolongo, P. L., \& Delgado, C. (2006). La revolución contemporánea del saber y la complejidad social: Hacia unas ciencias sociales de nuevo tipo. Buenos Aires: Clacso.

Tedesco, J.C. (2000). Educar en la sociedad del conocimiento. Buenos Aires: Fondo de Cultura Económica.

Valera, R. (2008). Acercamiento a la problemática actual de los estudios sociales. Curso: Participación Ciudadana y Educación Popular: confluencias y desafíos en el espacio asociativo latinoamericano/ Cátedra: Florestán Fernández /Aula 601/ Clase 1-/Clacso. Educación Virtual.

Vasilachis, I. (1993). Métodos cualitativos I: Los problemas teórico-epistemológicos. Buenos Aires: Centro Editor de América Latina.

Wallerstein, I. (2004). Las Incertidumbres del saber. Barcelona: Gedisa.

Zemelman, H. (2002). Pensar teórico y pensar epistémico: Los retos de las ciencias sociales latinoamericanas. Instituto Pensamiento y Cultura en América A. C., México. Recuperado de http://www.ipecal.edu.mx/Biblioteca/ Documentos/Documento7.pdf 